\title{
LA PHOTOGRAPHIE AU REGARD DES THÉORIES DE LA CULTURE ET DE LA COMMUNICATION
}

\author{
Jan Baetens et Gérard Derèze ${ }^{1}$
}

La photographie, tant le média que les pratiques qui le façonnent, est de nouveau partout. Contrairement à ce qui pouvait encore se dire ou se lire il y a dix ou quinze ans, la révolution technologique qui a secoué, puis presque effacé la photographie traditionnelle sur pellicule, n'a nullement signifié la disparition de la photographie elle-même. Après la mutation numérique, le plus ancien des nouveaux médias n'a pas été relégué au cabinet des curiosités historiques, même si, bien entendu, la photographie d'aujourd'hui n'est plus celle d'hier².

Or cette présence, ce dynamisme et les nouveaux défis du média photographique sont tout sauf étrangers à aux aspects que la numérisation semblait dans un premier temps avoir condamnés à l'obsolescence.

1 Respectivement, professeur à la KUL et professeur à l'UCL.

2 Tout comme, il est bon de le rappeler parfois, celle d'hier n'était déjà plus celle d'avant-hier : l'histoire de la photographie est une longue suite de ruptures et de relances, dont l'étude reste plus qu'utile pour qui veut nuancer un peu les réactions vives et alarmistes à chaque nouvelle transformation.

Recherches en communication, $\mathrm{n}^{\circ} 27$ (2007). 
Dans la presse, pour commencer, la photographie connaît actuellement un regain d'intérêt remarquable, en dépit du soupçon que les possibilités de manipulation digitale jettent sur l'authenticité de n'importe quelle image. S'il est vrai que le public a cessé de croire à l'innocence du document photographique, l'image même n'a rien perdu de sa force ni de son impact, comme l'a démontré de façon convaincante l'affaire des images d'Abou-Ghraib. De même, et davantage encore, l'avènement des nouvelles technologies a rendu possible l'invention de nouvelles formes de reportage, dont celles qui bouleversent les rapports entre ceux qui «font » et ceux qui « reçoivent» les photographies : le "journalisme citoyen", c'est-à-dire les nouvelles formes de participation privée à la production de l'information collective (par exemple à travers les photos prises sur les GSM des témoins directs d'un événement, puis divulguées sur des sites web, enfin relayées par les organismes de presse traditionnels), transforme le « consommateur » en «producteur », sans pour autant éliminer les questions relatives à la circulation de l'information'.

Dans la photographie d'amateur, la numérisation ne s'est pas traduite par le « saut » d'un média ancien, réputé moins performatif, à un média plus nouveau, jugé plus efficace en termes de réalisme et de pouvoir émotif -par exemple par le saut de l'image fixe de la photographie à l'image mobile de la vidéo. À la différence de ce qu'aurait pu suggérer le « grand récit» de la théorie de la remédiation de Bolter et Grusin ${ }^{2}$, la photographie résiste fort bien aux assauts des nouveaux médias. À tel point que, de plus en plus, c'est elle qui occupe le centre des réflexions théoriques sur l'image, non pas du point de vue technologique, mais du point de vue des usages sociaux qui s'infèrent de cette « chose » au carrefour de la culture et de la technologie. L'explosion des études photographiques -un secteur actuellement bien plus riche et vivant que celui des études sur la vidéo, par exemple- en est un symptôme fort significatif.

1 Voir André GUNTHERT, "Les photographies de l'EHESS et le 'journalisme citoyen"”, Études photographiques, n 18, 2006, pp. 120-137, et “L'image parasite. Après le journalisme citoyen", Études photographiques, n 20, 2007, pp. 174-186.

2 J. D. BOLTER ET R. GRUSIN, Remediation. Understanding New Media, Cambridge, Mass.: MIT Press, 1999. 
Du côté de l'esthétique, qui demeure un axe certes ambivalent mais central des recherches sur l'image photographique, l'apparition du numérique a permis aussi le renouvellement de bien des débats qu'on avait pu juger dépassés. Ici aussi, ces nouvelles questions touchent aux rapports entre producteurs et récepteurs. La vogue de la photographie trouvée, par exemple, explore et réexamine, sur de nouvelles bases, la hantise très ancienne de l'aspect "inhumain », c'est-à-dire purement mécanique, sans intervention de l'homme ${ }^{1}$. La question de la photo trouvée se pose en des termes spécifiquement photographiques, comme l'indiquent assez les différences de traitement entre l'objet trouvé dans les arts visuels (que l'objet en question soit ou non présenté selon la poétique du readymade), au cinéma (où le concept de found footage n'est pas dissociable de nouvelles pratiques de montage) et en photographie (où il sert entre autres à critiquer radicalement la coupure entre photo d'art et photo d'amateur, une très vieille question en théorie photographique). Corollairement, la numérisation a accéléré non moins les nouvelles approches de quelques thèmes devenus classiques de la critique photographique, tels que l'idée benjaminienne de la perte d'aura, qu'il est impossible de continuer à envisager de la même façon avant et après la numérisation -et que la numérisation oblige à réinterroger de façon critique pour l'ère analogique.

Quatrièmement -et sans que la présente énumération puisse être considérée comme exhaustive-, la numérisation a brouillé aussi bien des idées établies sur l'utilisation des images à l'intérieur des pratiques scientifiques. D'une part, l'expansion incessante des possibilités techniques du média a fait passer la photographie du statut d'adjuvant à celui d'objet proprement dit : l'image ne sert plus à étayer une recherche, la recherche sert au contraire à produire de nouvelles images. D'autre part, les propriétés complexes et très variées de ces nouvelles images scientifiques ont mis aussi en discussion bien des convictions fermement arrêtées sur ce qu'il convient d'inclure ou au contraire d'exclure du corpus d'images pouvant être étudiées par les sciences humaines qui s'intéressent d'une façon ou d'une autre au visuel, de l'histoire à l'esthétique, de l'iconologie à la philosophie² .

1 Cette perception de l'image acheiropoétique est bien sûr un mythe, comme l'a fort bien montré Fr. BRUNET dans son livre L'invention de l'idée de photographie, Paris, PUF, 2002.

2 Sur ces questions, voir J. ELKINS, Visual Studies. A Skeptical Introduction, Londres 
Ces quatre champs qui, avec bien d'autres, sont actuellement en train de réarticuler notre réflexion sur le média photographique (et le paysage médiatique plus vaste dont il fait partie et qu'il contribue à refaçonner activement à son tour) n'ont pas seulement en commun de refuser les clivages traditionnels des études photographiques (photo d'art versus photo d'amateur, fiction versus document, image authentique versus image manipulée...). Partout s'observe un même glissement d'une approche du média comme « énoncé » (objet ou forme) à une approche du média comme « énonciation » (acte, pratique, usage), bref à une approche qui s'interroge en tout premier lieu, non pas sur le sens d'une image, mais sur la manière dont le sens est construit, dans un processus de négociation permanente entre injonctions liées aux structures de production (dont les récepteurs font désormais partie) et modalités d'appropriation (qui sont inséparables de la manière, notamment technologique, dont les images se constituent).

Par définition inachevée et inachevable, cette articulation complexe, d'une donnée formelle -la photo comme image- et d'une série d'usages -la photo comme pratique sociale et comme instrument de production de sens- est au centre de toutes les contributions réunies dans ce numéro de Recherches en communication. Issues d'un colloque bilingue qui s'est tenu à Leuven et à Louvain-la-Neuve du 30 mai au 2 juin $2007^{1}$, les analyses et réflexions, que nous présentons en ces pages, partagent sans exception, tout en l'illustrant de façon très variée, l'idée fondamentale que la photographie est un outil de communication et de production de sens. Tournant le dos à des méthodologies qui se proposent de lire l'image photographique en elle-même -mais sans dire pour autant que de telles méthodologies ne seraient pas légitimes en d'autres domaines-, les auteurs de ce numéro insistent sur l'interaction entre l'image et son contexte, et ce dans les deux sens : car si le contexte modifie inévitablement le sens d'une image, le fonctionnement d'une image dans un contexte donné tire toujours à conséquence pour ce dernier, qui peut s'en trouver modifié lui aussi.

et New York, Routledge, 2003.

1 Pour plus de détails sur le programme complet de ces quatre journées, voir: http:// www.lievengevaertcentre.be/pdfs/cultureandcommunication.pdf 
Afin de permettre une meilleure lisibilité des enjeux théoriques et critiques de notre projet, les textes ont été regroupés en fonction des quatre grands axes distingués ci-dessus. Il importe toutefois de souligner que plusieurs articles abordent simultanément plusieurs de ces champs -ce qui prouve une fois de plus la grande proximité des questions qui se profilent à l'intérieur de chacun d'eux : on n'aborde pas forcément différemment la question du sens artistique et la question du sens journalistique à partir du moment où l'on part de la primauté du fonctionnement social, contextuel et historique du média.

Le texte de Paul Vancassel qui ouvre le numéro réunit de façon exemplaire la plupart des interrogations critiques et théoriques que supposent les nouvelles façons de penser l'outil et la pratique photographiques. S'appuyant sur une anthropologie des faits visuels, il propose ici une vue d'ensemble qui se propose à la fois d'élargir la palette théorique des études photographiques et de jeter des ponts entre plusieurs disciplines et traditions de recherche jusqu'ici ici assez décloisonnées. Les contributions de Catherine Saouter et de Maria Giulia Dondero mêlent les réflexions théoriques aux considérations pratiques et critiques. Ces textes ouvrent, chacun à leur façon, de nouvelles voies de la sémiotique de la photographie.

L'étude du discours journalistique est présente ici par deux approches portant sur des corpus peu étudiés, si ce n'est franchement négligés. Éric Pedon examine les transformations des grandes agences de presse, américaines aussi bien que françaises, de l'après-guerre à l'époque actuelle. Son étude ne met pas uniquement l'accent sur la manière dont changent les images, mais aussi et surtout sur les éléments qui déterminent ces changements, du statut de l'auteur aux nouveaux rapports entre texte et image. Analysant la rencontre du média photographique et de la blogosphère numérique, Sandro Faes explore les frontières internes des pratiques photographiques dont il n'est plus possible de dire si elles relèvent du document ou de la création.

Le domaine apparemment mieux balisé des études esthétiques de la photographie regroupe quatre contributions qui, elles aussi, réinterrogent, à bien des égards, les catégories fixes des historiens. L'article de Steven Humblet, qui aborde une image "oubliée" d'une photographe par ailleurs tout à fait célèbre, Berenice Abbott, porte cette suspicion à un degré supérieur dans la mesure où l'auteur accède aussi au dossier 
"caché" (dans une archive new-yorkaise) de la genèse de l'image scrutée. Michel Frizot, quant à lui, présente une analyse d'un corpus de photographies anonymes, qui se transforme rapidement en une véritable esthétique de ce type d'images. L'étude de Danièle Méaux, sans doute la plus littéraire de ce numéro, introduit une problématique de nos jours capitale pour les écrivains, celle de la contrainte ou règle préconstruite (au sens oulipien du terme), pour l'appliquer à un corpus où on ne l'attend guère, celui des livres photographiques de voyage. Enfin, Alexandre Streitberger fait très utilement le point sur les manières dont le monde de l'art s'approprie le média photographique, notamment dans les techniques d'exposition qui font de nos jours partie intégrante de l'œuvre même.

Enfin, la diversité des usages scientifiques de la photographie ressort bien des deux articles qui terminent ce dossier et qui montrent les deux grandes voies qu'a toujours empruntées la démarche scientifique dans ses contacts avec la photographie. La photographie comme objet d'analyse est au cœur de l'article de Laurence Van Ypersele, qui commente les problèmes spécifiques que pose le recours à l'image à une discipline qui avait longtemps réduit la notion de source aux seuls documents écrits ou oraux. La photographie comme outil, mais aussi comme but d'analyse est illustrée par l'étude de Cécile Tardy, qui présente les techniques et les enjeux de la photographie géographique documentaire. Les images qui résultent des procédures hypercontraintes qu'elle décrit ne sont toutefois pas sans susciter des échos du côté de l'art, où l'on sait l'importance des recensements sériels, ou du côté du reportage, qui réutilise parfois dans de tout autres perspectives les images faites dans des situations de laboratoire. 